Ч્પ FRANÇAISE

$\supset$ DE

딜 PEAGOGIE

\section{Revue française de pédagogie}

Recherches en éducation

155 | avril-juin 2006

La motivation scolaire : approches récentes et perspectives pratiques

\title{
Professeurs des écoles novices : formes collectives et individuelles du " passage à travers le miroir »
}

Inexperienced elementary school teachers: collective and individual forms of "passing through the looking glass"

Profesores de escuela novatos: formas colectivas e individuales del "paso por el espejo"

Grundschullehrer als Anfänger: kollektive und individuelle Formen des

"Übergangs durch den Spiegel“

Patrick Dubois, Rachel Gasparini et Gérard Petit

\section{OpenEdition}

Édition électronique

URL : http://journals.openedition.org/rfp/232

DOI : $10.4000 /$ rfp.232

ISSN : 2105-2913

Éditeur

ENS Éditions

Édition imprimée

Date de publication : 1 juin 2006

Pagination : 73-82

ISBN : 978-2-7342-1047-4

ISSN : 0556-7807

\section{Référence électronique}

Patrick Dubois, Rachel Gasparini et Gérard Petit, «Professeurs des écoles novices : formes collectives et individuelles du «passage à travers le miroir » », Revue française de pédagogie [En ligne], 155 | avriljuin 2006, mis en ligne le 21 septembre 2010, consulté le 19 avril 2019. URL : http:// journals.openedition.org/rfp/232 ; DOI : 10.4000/rfp.232 


\section{Varia}

\section{Professeurs des écoles novices: formes collectives et individuelles du "passage à travers le miroir »}

Patrick Dubois, Rachel Gasparini, Gérard Petit

Les professeurs des écoles novices éprouvent en IUFM les tensions structurelles d'une formation professionnelle, qui leur paraît pour partie perpétuer un état de sujétion dans l'institution scolaire auquel ils veulent échapper au plus vite pour passer de l'“autre côté du miroir ", et accéder à une vie professionnelle qui est aussi souvent l'entrée dans le monde adulte. Beaucoup paraissent hésitants à s'impliquer dans certains dispositifs récents de cette formation, organisés autour du paradigme du "praticien réflexif ": soit qu'ils soient portés, par leur histoire scolaire/universitaire propre, à valoriser d'autres dimensions du métier, soit qu'ils rechignent à exposer publiquement leurs difficultés, dans la crainte des usages institutionne/s et sociaux de cet aveu, ou le pressentiment qu'il puisse accroitre leur vulnérabilité plutôt que la réduire.

Descripteurs (TEE) : apprentissage par la pratique, expérience de l'enseignement, IUFM, préparation des cours, professeur des écoles, professionnalisation.

$\mathrm{D}_{\mathrm{m}}^{\mathrm{e}}$ epuis la fin des années 1980, l'exercice du métier d'enseignant est communément défini, dans les textes officiels ou la littérature spécialisée, comme une mobilisation «professionnelle » de compétences multiples (1) : la maîtrise de savoirs théoriques, disciplinaires, didactiques, institutionnels, etc.; mais aussi, celle de compétences pratiques variées de conception et de conduite d'actions d'apprentissage en milieu scolaire, incluant l'aptitude à opérer des entorses aux parcours didactiques initialement programmés, à effectuer des arrêts impromptus sur difficulté, des rappels à l'ordre improvisés, des retours en arrière, des chemins de traverse, etc. ; corrélativement, la capacité à analyser sa pra- tique professionnelle dans la perspective de son amélioration (Lang, 1999 ; Perrenoud, 1999).

On connaît, par diverses études (Peyronie, 1998 ; Perrenoud, 2001 ; Blanchard-Laville, 2001 ; Dubet, 2002), les griefs que les professeurs d'école stagiaires émettent sur leur formation à cette professionnalité dans les IUFM. Ces travaux ont, entre autres, montré les limites des explications internes à l'institution, lorsqu'elles concluent à l'inadéquation conjoncturelle, quantitative et/ou qualitative, de l'offre de formation à la demande ou même, parfois, s'aventurent à déplorer l'« utilitarisme» des futurs maîtres - nouvel avatar des « incapables prétentieux » 
(Isambert-Jamati, 1985). Dans le premier cas, la constance des insatisfactions, en dépit des divers aménagements de la formation déjà opérés, suffit à fragiliser une analyse simplement indexée à l'idée d'une réforme en termes d' « un peu plus de »-un peu plus d'enseignements disciplinaires, didactiques, pédagogiques, etc. Dans le second, le niveau d'études des stagiaires et leur investissement fréquent, au cours de leur formation, dans des activités de conceptualisation - la planification de leur enseignement au cours des stages, la réalisation d'un mémoire professionnel, etc. - disqualifie les jugements sommaires mettant en cause leur refus ou leur incapacité du travail théorique.

Au-delà des critiques qu'ils apportent à leur formation, il nous est apparu important de vérifier dans quelle mesure les stagiaires s'approprient, d'emblée ou au cours de cette année de formation, ces thématiques de la "professionnalisation » du métier et les valeurs qui y sont attachées. Nous avons tenté de le faire à partir d'une enquête que nous avons effectuée en 2001-2002, auprès des soixante-dix-sept professeurs d'écoles de deuxième année (PE2) inscrits dans le même centre départemental d'lUFM (2).

\section{TENSIONS STRUCTURELLES D'UNE FORMATION AU MÉTIER}

Ce travail confirme les études antérieures : l'éloge de la «pratique » et la critique de la «théorie » font partie des discours attendus dans les formations à l'enseignement, d'autant que les savoirs de référence sur lesquels elles s'appuient souffrent d'un manque de légitimité, contrairement à d'autres, comme la médecine ou le droit (3). Plus précisément, l'enquête a mis en lumière une double tension. La première est interne à la formation professionnelle elle-même. L'institut de formation, en effet, à la fois soumis aux objectifs que lui assigne l'État employeur - lui présenter en fin d'année une population de novices aptes à la titularisation - et héritier des institutions qui l'ont précédé et plus généralement de l'histoire de l'école française, met en place une socialisation professionnelle dont les aspects «scolaires "lato sensu ne sont pas évacués : elle passe par une programmation sur l'année d'enseignements conformes à un cahier des charges officiel, des obligations institutionnelles imposées aux stagiaires - assiduité, ponctualité, etc. -, de nombreuses heures de cours où ils demeurent physiquement assujettis à une position d'“élèves ", selon la représentation traditionnelle qu'ils s'en donnent, où ils subissent des formes variées, plus ou moins vécues comme contraignantes, d'incitation à l'implication dans la formation et de contrôle de leurs acquisitions, etc. Rien n'indique pourtant qu'ils remettent en cause cette forme scolaire de leur formation initiale au métier, mais à coup sûr certaines tournures aiguës ou directives de celle-ci, moins liées au principe de leur formation qu'à sa mise en œuvre par les acteurs de l'institution.

Ce qu'ils dénoncent, en effet, c'est l'écart qu'ils ressentent entre les principes affichés dans le plan officiel de leur préparation au métier - «formation d'adultes " favorisant la responsabilisation du futur enseignant, son «projet personnel de formation", etc. - et la réalité de certaines pratiques sur le lieu de leur formation qui, s'autorisant des textes réglementaires qui encadrent celle-ci, leur paraissent démentir dans les faits ces principes, en les contraignant à des conduites de soumission aux attentes de l'institution. Cette tension s'exprime par exemple dans le discours qui leur est tenu par les agents de leur formation, qu'ils perçoivent comme paradoxal, à la fois collégial (marqué parfois par le tutoiement des stagiaires) et hiérarchique: d'une part, sur fond du mythe d'une communauté d'égaux associant formateurs et formés dans le même métier, l'autonomie du « collègue " novice et la responsabilité qu'il est invité à prendre dans sa formation, en oubliant son «métier d'élève» antérieur, sont exaltés; mais d'autre part, en contrepoint, un discours de « domination » peut à tout moment lui rappeler son état de sujétion dans l'institution, et le pouvoir que possède celle-ci de barrer la route à son recrutement dans la fonction publique, en ne validant pas son année de formation. Sans doute, ce discours de domination ne s'exprime-t-il ouvertement que dans les situations critiques de conflit entre un stagiaire et l'institution ; mais chaque membre de celle-ci peut toujours y recourir, selon la logique de son tempérament et le type de directivité qu'il impose aux stagiaires, sur un mode d'énonciation à sa discrétion - de l'allusion discrète à la menace explicite.

Mais selon les histoires personnelles, ce mode scolaire de socialisation professionnelle produit des effets variés, et parfois chez le même enseignant débutant, en fonction des contextes: souvent rejetée - selon des proportions variables d'un stagiaire à l'autre pour cause d'infantilisation ou d'inadaptation à un public d'adultes, cette formation trop "scolaire " est parfois recherchée pour le cadre rassurant qu'elle 
offre face aux incertitudes constitutives du métier d'enseignant et à l'engagement personnel qu'il appelle. Ainsi, l'enquête fait apparaître qu'un certain nombre d'enseignants débutants, tout aussi critiques que les autres à l'égard de leur formation, déplorent surtout qu'elle ne leur offre qu'insuffisamment ce bagage d'" outils", de «réponses", de séquences "prêtes à l'emploi ", etc., qu'il suffirait, en «bons élèves " et sur un mode très scolaire, d'appliquer ensuite sur le terrain. On peut supposer aussi que la demande d'une présence plus forte de formateurs de terrain, en lieu et place d'universitaires ou de formateurs issus du second degré, recouvre parfois la même attente.

Cette tension interne à la formation professionnelle d'enseignants n'est sans doute qu'une modalité particulière d'une tension plus générale traversant le mode scolaire de socialisation dans notre société contemporaine, où l'injonction permanente à l'autonomie et à la responsabilité, à l'école, au collège, au lycée, vient sans cesse buter sur les contraintes propres à la scolarisation: obligation de présence, assiduité, régularité, répétitivité des activités et de leur contrôle, etc. Or les enseignants novices, à la différence d'autres professionnels en formation, sont placés dans la situation particulière qu'ils se préparent à devenir eux-mêmes agents de la socialisation scolaire, à laquelle ils soumettront leurs propres élèves comme ils $y$ seront eux-mêmes soumis. Toutefois, les vives critiques qu'ils adressent à certains aspects de la formation qu'ils reçoivent ne les conduisent qu'exceptionnellement à une réflexion critique, par analogie, sur les effets possibles de leur propre enseignement.

II en est ainsi de leur appréhension des situations d'évaluation, qui pousse les stagiaires à la demande pressante d'un soutien attentif et bienveillant de la part de leurs formateurs. Elle ne les mène pourtant pas à l'hypothèse que cette inquiétude pourrait exister dans les mêmes termes et avec les mêmes effets psychologiques chez leurs propres élèves (ou certains d'entre eux) et qu'elle exigerait dès lors de l'enseignant un niveau élevé de disponibilité et d'ingéniosité didactique et pédagogique pour y répondre: ces qualités, en effet, n'apparaissent pratiquement jamais dans la représentation de l'enseignant qu'ils aimeraient être. Autrement dit, le discours officiel - et sa vulgate ambiante - sur l'élève au « centre des apprentissages » ne parait pas signifier encore pour les stagiaires en cours de formation que l'élève apprenant et ses difficultés spécifiques soient mis au centre de leurs préoccupations. La nécessité pour l'enseignant d'avoir le « contact » avec les élèves et d'être à «leur écoute », maintes fois soulignée dans la représentation qu'ils se font d'un enseignant idéal, ne va pas jusqu'à la reconnaissance chez l'élève d'appréhensions de même nature que celles qu'ils éprouvent, ni de l'urgence d'un comportement adapté à son égard, sur le modèle de celui qu'ils revendiquent pour eux-mêmes.

La seconde tension, qui renforce les effets de la première, est l'inadéquation entre le modèle officiel du «bon maître d'aujourd'hui » (Baillauquès \& Breuse, 1993) et les images que les enseignants débutants se font de leur métier. La formation dans les IUFM, depuis leur création en 1989, s'est appuyée sur un modèle de professionnalité intégrant des compétences «professionnelles » jugées désormais indispensables à l'exercice du métier dans le contexte complexe des missions qui y sont attachées (Bourdoncle, 1991, 1993 \& 2000 ; Lang, 1999 ; Peyronie, 1998). Parmi celles-ci :

- la capacité à résoudre des situations singulières complexes et à prendre des décisions dans l'incertitude ou l'urgence (Perrenoud, 2001): si le métier d'enseignant ne peut être simplement acquis "sur le tas ", les savoirs théoriques ou procéduraux transmis par enseignement ne suffisent pas à eux seuls à résoudre une partie importante des problèmes posés dans la pratique; aussi, cette formation, prenant en compte les recherches conduites, à la suite de Schön, sur le paradigme du "praticien réflexif", ambitionne d'équiper les stagiaires d'une posture réflexive habituelle à l'égard de leur travail et des conditions dans lesquelles il l'exercent.

- la prise en charge de l'« hétérogénéité " des classes: les valeurs altruistes qui sous-tendent la formation en IUFM sont en continuité avec celles qui inspiraient la formation des écoles normales, de démocratisation de l'accès aux savoirs et de lutte contre la «mortalité » scolaire. Mais elles s'expriment dans un discours neuf et neutre de maîtrise de classes « hétérogènes », pour lesquelles une pédagogie est officiellement préconisée, à la fois " active" - « constructiviste» - et « différenciée", visant à rendre les élèves « acteurs de leurs apprentissages " et l'enseignement ajusté aux difficultés individuelles.

Désormais intégré aux textes officiels relatifs à la formation des enseignants, ce modèle de professionnalité ne se greffe probablement pas sans difficulté sur les représentations propres qu'ont du métier les enseignants novices, dont l'imaginaire de 
la profession - souvent enchanté - s'est nourri et cristallisé tout au long de leur histoire scolaire. En effet, parmi les qualités qu'ils déclinent, dans notre enquête, de l'enseignant qu'ils souhaiteraient être, c'est la dimension personnelle (expressive et relationnelle) qui est fortement mise en avant, en début comme en fin d'année. Ce modèle charismatique du métier d'enseignant, qui s'est construit au cours de leur histoire scolaire, primaire et secondaire, voire supérieure, résiste donc, chez la majorité d'entre eux, aux représentations concurrentes que leur renvoie la formation initiale.

Or, ces qualités qu'ils jugent primordiales - la "personnalité ", le bon contact avec les enfants, l'autorité, la patience, le dynamisme, l'aisance dans la classe, etc. - sont en outre celles qu'ils jugent ne pas relever d'une formation professionnelle, au motif qu'elles sont constitutives de la personne ellemême. Elles n'ont besoin que d'être complétées par des savoir-faire pratiques, qu'ils sont convaincus pouvoir acquérir essentiellement sur le terrain et dans le contact avec des hommes et femmes de métier.

Une telle représentation du métier (sa diffusion et sa résistance à la formation) s'explique par plusieurs raisons :

- elle est beaucoup moins déstabilisante que le modèle du "praticien réflexif ", puisqu'elle pense le métier comme une somme stable de savoir-faire de base - tenir une classe, transmettre un programme, évaluer les acquis - davantage que comme une remise en question permanente dans un océan d'incertitudes;

- elle est congruente aux images enchantées du métier que les stagiaires tirent de leur propre passé scolaire (qui ont parfois été à la source de leur choix professionnel) ; elle fait donc l'économie du "passage à travers le miroir" (Hughes, 1996), de cette conversion identitaire qui impose de «voir le monde à l'envers" - les élèves, le matériel scolaire, les contraintes institutionnelles, mais aussi l'enseignant lui-même -, avec les yeux de l'enseignant et non plus ceux de l'ancien écolier ;

- elle offre des bénéfices narcissiques, mettant au centre l'enseignant et sa "personnalité ", ce noyau de qualités auxquels il suffit d'adjoindre les « tours de main " pratiques pour exercer valablement le métier ;

- elle est confirmée par le discours "sécurisant" de nombreux collègues de terrain: "les programmes passent, le métier reste... tout ça c'est de la théorie ; le métier, c'est autre chose... »
Corrélativement, peut-être, assez peu de stagiaires incorporent à l'image du "bon maître " son engagement pour les valeurs que revendiquaient leurs aînés - dont certains sont aujourd'hui leurs formateurs -, la démocratisation de l'enseignement et la lutte contre l'échec scolaire, notamment des élèves socialement ou culturellement défavorisés. Ils ne sont certes pas tous insensibles aux différences et aux inégalités entre les élèves, mais lorsqu'ils expriment ce souci - un quart des stagiaires en début d'année, et seulement un cinquième en fin d'année c'est plutôt d'une manière générale, comme le désir d'un enseignement au service de tous, adaptable aux différences individuelles, "gérant l'hétérogénéité " (4). En cela ils ne diffèrent guère des jeunes professeurs du secondaire interrogés par $P$. Rayou et A. van Zanten (2004). Mais comme eux aussi, ils ont peut-être tellement intégré les analyses relatives au rôle de l'école dans la reproduction des stratifications sociales, qu'ils éprouvent probablement moins que leurs aînés le besoin de les évoquer dans des professions de foi générales et incantatoires, pour adopter une vision plus pragmatique et individualisée des difficultés scolaires des élèves, ici et maintenant.

Cela dit, on n'ignore pas que l'affirmation de la "prise en compte de l'hétérogénéité", qui fait désormais partie de la vulgate ambiante dans le monde enseignant, relève parfois davantage d'une posture de principe que de pratiques réelles, tant celles-ci sont inévitablement complexes, mobilisent souvent une grande ingéniosité de la part de l'enseignant et offrent rarement des résultats à très court terme (Davisse \& Rochex, 1997; Barrère, 2003). D'une manière générale, on sait que les méthodes traditionnelles (pédagogie frontale, récitation, etc.) sont encore très présentes à l'école primaire (Dubet, 2002), que le mode expositif-interrogatif est plus utilisé que ne le laisseraient à penser les discours sur "l'enfant acteur" ou la "socio-construction" des savoirs (Altet, 1994). A fortiori toutes les pratiques recommandées pour différencier la pédagogie en fonction de la diversité des élèves posent inévitablement de réels problèmes aux jeunes stagiaires.

Sans doute, parmi ceux-ci, des différences plus ou moins sensibles sont toujours perceptibles. Ainsi, l'importance attribuée aux savoir-faire professionnels par rapport à ce qui est indexé au noyau dur de la "personnalité » est un facteur probablement non négligeable de la disponibilité du stagiaire à l'analyse réflexive de sa pratique. Toutefois, c'est cette inadéquation forte des représentations du métier aux 
attentes de la formation qui paraît au principe de la disqualification par nombre d'entre eux des enseignements "théoriques" au profit de l'expérience apportée par le terrain. Elle les conduit aussi à durcir leurs représentations de la formation, méconnaissant à la fois la complexité de l'apprentissage en centre et ce qui se passe effectivement sur le terrain. Dans le centre de formation, en effet, la " forme scolaire » d'apprentissage (Vincent, 1980 \& 1994) ne se limite pas à la diffusion sous une forme magistrale de savoirs formels, validés scientifiquement ou non: nombre de formateurs en salle évoquent des expériences de terrain, ont recours à des exemples, voire des anecdotes pour illustrer leurs propos, évoquent des «trucs » ou « astuces » de métier, donnent des exemples de séquences/séances, montrent des vidéos de classes en situation, etc. Inversement, l'apprentissage de terrain n'est pas complètement étranger à des références théoriques au moins implicites, ni au mode scolaire d'apprentissage (par exemple, la planification des séquences d'enseignement est organisée en mobilisant des savoirs théoriques divers, avec des objectifs rationnels, tels ceux d'aménager une progression logique en lien avec les programmes, de prendre en compte le niveau des enfants; elle est reprise et amendée à partir de bilans réflexifs réguliers sur les interactions dans la classe, etc.).

\section{LE TERRAIN : CONFLIT DES INTERPRÉTATIONS}

Pour l'institut qui accueille les enseignants novices, la formation vise officiellement à construire des compétences professionnelles à partir d'un dispositif " en alternance ", combinant des temps de formation au centre et des temps de pratique professionnelle contrôlée. Ceux-ci doivent équiper le stagiaire d'un premier "savoir pratique », ce «fourre-tout indispensable " (Malglaive, 1990) qui prend en charge tout ce que les savoirs théoriques et procéduraux laissent dans l'ombre; ils valent aussi comme moment d'épreuve du réel, à partir duquel les futurs enseignants exerceront leur capacité d'analyse réflexive, à leur retour au centre de formation.

Mais pour beaucoup d'entre eux, les choses n'en vont pas tout à fait ainsi : les stages de terrain représentent aussi - voire d'abord - l'occasion d'échapper pendant quelques semaines à la pression des formes les plus directives du mode scolaire de socialisation imposé par l'institut, de vivre enfin le métier pour lequel ils se préparent, et d'entrer en relation avec des collègues étrangers à l'institut. Certes, ils n'abordent pas avec insouciance cette prise de responsabilité sur le terrain. Les premières confrontations avec la classe sont souvent redoutées. Exprimée directement ou non, c'est la hantise de ne pas y arriver, de se faire déborder ou d'être trop autoritaire, de ne pas savoir préparer efficacement son travail, de ne pas arriver à faire entendre son point de vue face à l'enseignant titulaire ou à l'équipe de formateurs assurant le suivi pédagogique des stages, etc. Une expression revient souvent dans l'enquête, celle de " ne pas être à la hauteur " (5), qui traduit cette peur diffuse d'échec, relative aussi bien au contexte du terrain d'exercice (6) qu'au jugement porté par les formateurs chargés d'évaluer leur prestation en classe (7). Deux inquiétudes, toutefois, sont plus rarement formulées. La première se rapporte à la personnalité même du stagiaire, à ses réactions dans certaines situations critiques (8); la seconde, aux effets de ses éventuelles maladresses pédagogiques sur les apprentissages des élèves (9).

Ce faible taux de réponses relatives aux effets de l'enseignement sur les élèves ne signifie pas qu'ils soient méconnus des stagiaires, mais plutôt, probablement, que les appréhensions relatives aux situations professionnelles pénibles sont plus spontanément rapportées à eux-mêmes (au malaise qu'ils pourraient en éprouver) qu'à leurs effets pédagogiques. D'une manière générale, en effet, il semble bien que les difficultés redoutées - et indirectement les critiques adressées à la formation théorique, qui prémunirait insuffisamment contre elles - renvoient nettement plus aux prestations des stagiaires - « ne pas arriver à faire ce que j'avais prévu »- qu'aux conséquences sur les apprentissages des élèves, notamment ceux qui sont en difficulté. On retrouve ici en creux le modèle idéalisé du "bon maître ", construit en partie dans la socialisation antérieure du sujet (lycée, université), dont l'efficacité serait principalement fonction de la validité des savoirs transmis, des qualités expressives de l'enseignant et de l'«attention" des élèves. On peut penser que ce sont les effets de ce modèle incorporé qui conduisent nombre de stagiaires tout à la fois à sur-valoriser les situations d'évaluation qu'ils subissent au cours de leur stage - un jugement sur leur personne propre - et, non sans paradoxe peut-être, à atténuer d'autant leur vigilance à l'égard des conséquences sur les élèves de leurs actes pédagogiques.

Mais le temps de stage leur donne aussi l'occasion de rencontrer d'autres professionnels que les forma- 
teurs de l'IUFM, avec lesquels ils peuvent espérer entrer en contact en toute liberté. Cette collégialité de terrain, vécue comme plus "authentique "que celle - en «trompe l'œil »- qu'ils dénigrent dans le centre de formation, est appréciée pour la "régulation entre pairs " (Rayou \& van Zanten, 2004) qu'elle autorise, l'apport de menus conseils ou encouragements permettant des ajustements au quotidien. Ils n'hésitent pas à solliciter cette assistance ponctuelle de proximité, dans la mesure où, d'un maître à l'autre, tout au moins dans un même cycle, les tâches prescrites paraissent très semblables (à la différence de ce que vivent les jeunes professeurs stagiaires des lycées et collèges); et ils en sont d'autant plus friands que l'activité en solitaire dans une classe avec un groupe d'élèves, à laquelle tiennent plus que tout de nombreux maîtres titulaires, pour l'indépendance professionnelle qu'elle procure, est vécue par eux comme éprouvante, confrontés comme ils le sont, sans le filet de protection de l'expérience, aux innombrables micro-décisions du quotidien. Les maîtres en poste, dans leur majorité, semblent se prêter à cet accompagnement collégial, dès lors qu'il ne leur paraît pas une ingérence sur leur territoire propre.

Or, ces expériences et acquisitions informelles (10), qui donnent du sens à ce qu'ils vivent sur le terrain, peuvent conforter ou à l'inverse brouiller, parasiter le discours professionnel délivré par l'institution. Et celui-ci n'est pas assuré de sortir gagnant d'une confrontation avec les représentations et valeurs des enseignants en poste, étant donné la légitimité dont ils jouissent, du fait de leur maîtrise des "ficelles " du métier, et de ne pas apparaître comme irréel, au regard de leur "réalisme" professionnel (11). Mais ce conflit, s'il a lieu, atteste aussi que le "terrain " est socialisateur, fabricateur d'identité professionnelle autant que la formation en institut, par la transmission informelle de normes et de valeurs sur le "bon" exercice du métier, que le stagiaire peut s'approprier autant que les savoirs et les injonctions à la réflexivité de l'institution (12).

Car sur le terrain, les attentes collégiales sont d'une autre nature que celle de l'institut de formation. Le stagiaire n'est pas apprécié à l'aune du paradigme du "praticien réflexif ", mais à proportion de son respect de certains codes explicites ou implicites : celui de la réglementation officielle (ponctualité, règles de sécurité, contrôle des absences, respect du tableau des tours de rôle de surveillance de récréation, etc.) ; celui, aussi, de certaines lois coutumières propres au lieu d'exercice (ne pas faire de zèle par un respect strict de l'horaire officiel des récréations, si l'horaire coutumier excède la durée légale, etc.) ; l'adoption d'une posture discrète, enfin (pas de valorisation déplacée des savoirs théoriques acquis à l'IUFM).

\section{L'INJONCTION RÉFLEXIVE ET SES LIMITES}

Depuis les travaux pionniers de Schön, dans les années 1980-1990, on sait que de nombreuses recherches, notamment dans le secteur des métiers de l'interaction humaine, ont conforté la conviction que la pratique professionnelle n'y était pas réductible à l'application des théories élaborées en dehors d'elle, mais qu'elle constituait elle-même un lieu de production de savoirs "en acte». A leur suite, le large consensus dont jouit désormais en France le modèle du "praticien réflexif " dans les milieux de formation des enseignants (Perrenoud, 2001 ; Lang, 1999), a conduit à valoriser, dans la formation initiale de ceux-ci, les temps de "réflexion sur l'action", avec le postulat implicite qu'ils amorceraient la construction identitaire d'un professionnel capable, selon l'expression de Schön (1983), de "reflexion in action ", d'autorégulations dans l'urgence de l'action (13). Nous avons suggéré précédemment qu'il n'était pas sûr que ce paradigme nouveau du métier prenne aisement place dans les representations que les enseignants novices se font de celui-ci. Dans notre échantillon, lorsqu'ils décrivent l'enseignant qu'ils aimeraient être, seuls trois d'entre eux (sur soixantedix-sept) mentionnent l'aptitude à réfléchir sur leurs pratiques ou à se remettre en question; en fin d'année, ils ne sont que dix (sur soixante-dix-sept) à reprendre l'item (14). Sans doute cette réserve à l'égard d'un modèle professionnel pourtant constamment invoqué en formation - au risque parfois de s'égarer dans une rhétorique incantatoire peut-elle en partie s'expliquer par les procédures mises en œuvre, dans le contexte étudié, qui paraissent principalement ordonnées à un « paradigme de l'expertise » (15), peu congruent aux situations d'incertitude vécues par les stagiaires, ni peut-être véritablement approprié à la construction, en formation initiale, de premières compétences d'autorégulation rapide dans l'action. Mais elle nous semble résulter aussi de la focalisation durable des stagiaires sur d'autres dimensions de la profession que les compétences réflexives, qui exercent sur eux une forte séduction, comme les routines d'action qu'ils perçoivent chez leurs aînés, tant elles leur semblent 
constitutives de l'aisance qu'ils admirent chez eux et à laquelle ils aspirent vivement.

Il y a plus, probablement. Intervenant ici essentiellement après l'action, sous la forme d'une analyse des situations d'enseignement vécues en classe, dans une visée d'amélioration des pratiques par le biais d'essais de formalisation de l'expérience, ces moments réflexifs ne sont pas sans effets sur l'image de soi du futur professionnel; ils se présentent d'ailleurs aussi avec l'objectif d'une lucidité plus grande sur soi-même, dans son comportement professionnel et à travers lui, d'un rapport privé de soi à soi plus clairvoyant pour mieux faire face à la complexité du métier. Toutefois, cette capacité réflexive ne peut commencer à se développer dans l'institution de formation qu'en s'objectivant devant témoins, en se publicisant, en quelque sorte, au travers de diverses procédures codifiées, telles que les séances de préparation du mémoire professionnel, les rencontres régulières avec l'équipe de formateurs au moment des stages, les moments d'analyse collective des pratiques de chacun, etc. Or, dès lors qu'ils savent que cette analyse peut devenir une pièce au dossier de l'évaluation globale de leur formation, les stagiaires redoutent fortement, bien audelà des risques réels qu'ils encourent, ces moments où ils sont incités à exposer - aux deux sens du terme - leur pratique. D'autant que, dans le récit ainsi rendu public d'expériences parfois très personnelles, le référentiel de compétences, qui fixe pour tous les membres de l'institution ce que l'on est en droit d'attendre des stagiaires en formation, n'est probablement plus le seul outil d'évaluation du stagiaire. D'autres normes sociales plus implicites entrent en jeu, relatives à l'" ordre scolaire " et ce qu'il doit être, au bruit tolérable dans une classe, au soin du travail de l'enseignant, à la «correction » de sa présentation de soi (habillement, maquillage, manière d'être, etc.) et de son langage, etc. (16).

En outre, la construction d'une posture réflexive en formation initiale s'adresse à des novices, par définition dépourvus du filet protecteur d'un habitus professionnel permettant de puiser des ressources didactiques ou pédagogiques pour rebondir devant l'échec, voire de s'y résigner. Dès lors, l'analyse réflexive appliquée à des situations critiques ou mal vécues peut conduire assez vite un sujet fragilisé à mettre directement en cause son image de soi, réactiver des angoisses, ébranler une confiance en soi vulnérable. On s'approche alors de zones sensibles où des désirs enfouis - d'être aimé, de dominer, etc. - pourraient faire surface et générer des souf- frances que l'institution, dans son fonctionnement ordinaire, ne peut prendre en charge. Enfin, si l'échec est bien le moteur d'une prise de conscience réflexive (Vermersch 1994 ; Perrenoud, 2001), on peut s'interroger sur la possibilité d'une analyse réflexive résultant non pas tant d'un écart personnellement ressenti entre la réussite visée et l'action effective, que d'un désaccord entre la perception qu'a le stagiaire de ses actes professionnels et celle que lui renvoie l'institution. Aussi, face aux divers risques que pourrait représenter cet usage public de la réflexivité dans le cadre de la formation IUFM, les stagiaires ont appris à se protéger. Quelques-uns ont l'habileté de mettre en avant des difficultés mineures pour occulter des problèmes plus profonds (car ne pas trouver de "problèmes" dans l'évaluation de ses pratiques devant des formateurs pourrait paraître suspect); d'autres reproduisent artificiellement le langage officiel du «praticien réflexif », sans en adopter véritablement la posture.

\section{CONCLUSION}

Les réflexions qui précèdent proposent quelques éléments d'analyse relatifs à ce qui se joue derrière les réticences et résistances des enseignants stagiaires à l'égard de leur formation en IUFM. Beaucoup d'entre eux arrivent en formation desireux de passer au plus vite "de l'autre côté du miroir ", dans une vie professionnelle qui les fasse enfin entrer dans le monde adulte. Aussi réagissent-ils, parfois vivement, contre les formes les plus directives du mode scolaire de socialisation en IUFM. Dans le même temps, ils entrent en formation porteurs d'un habitus scolaire-universitaire - dont, au passage, ils ignorent le plus souvent l'existence - qui les conduit à désirer durablement des modèles du «bon enseignant " qui ne sont pas toujours congruents avec celui autour duquel leur formation est organisée, notamment le paradigme du « praticien réflexif $»$. On pourrait incriminer les diverses formes de regimbement qu'ils expriment au nom de la rationalité de ce modèle et de l'archaïsme des figures de professionnalité auxquelles eux-mêmes demeurent attachés. À moins que l'injonction actuelle à la réflexivité dans la formation des enseignants ne soit qu'une déclinaison particulière d'un processus culturel beaucoup plus global d'«intellectualisation croissante du monde social " (Martuccelli, 2002) ? Avec une face claire, cette expansion de la réflexivité, "fille aînée des Lumières", qui développe chez les sujets un 
regard critique de soi à soi, mettant à mal toute imagerie naïve, "substantive » de la subjectivité; et peut-être une face sombre, si l'on suit jusqu'au bout les analyses de Foucault (17) (1975), selon lesquelles ces injonctions à l'analyse par le sujet de ses actes et de ses conséquences, qui, en cas de difficultés, le confronte devant témoins (pairs ou formateurs) à ses propres limites, relèveraient de procédures subtiles d'assujettissement (avatar laïcisé de techniques culpabilisantes de confession).

Cette perspective critique, dans sa radicalité, peut-elle aider à penser les processus mis en œuvre dans la formation des professeurs des écoles? Elle nous paraît a minima inviter à une vigilance prudente: la "culture psychothérapeutique " ambiante (Martuccelli, 2002) octroie a priori une telle évidence aux "techniques" de réflexion sur soi visant à accroître l'efficacité des actes du sujet, que des usages multiformes de celles-ci peuvent aisément trouver place dans les pratiques institutionnelles, au nom de l'« analyse des pratiques"; certes, nous savons que des protocoles rigoureux d'analyse des pratiques existent, qui permettent aux enseignants novices, comme aux autres, de réfléchir méthodi- quement sur la signification de leurs actes professionnels sans courir le risque que ce qu'ils en révèlent à autrui se retourne contre eux ; ils sont toutefois peu économiques (formation par petits groupes, animateur ne participant pas à l'évaluation des stagiaires, etc.), donc limités et d'avenir incertain, dans une logique industrielle de la formation des enseignants.

Patrick Dubois patrick.dubois@dijon.iufm.fr

IUFM de Bourgogne

\& Unité mixte de recherche «Éducation \& politiques" (INRP - université Lumière-Lyon 2)

Rachel Gasparini rachel.gasparini@univ-lyon2.fr IUFM de Lyon Groupe de recherche sur la socialisation (CNRS - ENSLSH - université Lumière-Lyon 2) \& Unité mixte de recherche "Éducation \& politiques" (INRP - université Lumière-Lyon 2)

Gérard Petit gerard.petit@dijon.iufm.fr IUFM de Bourgogne.

\section{NOTES}

(1) Elles sont par exemple, pour les professeurs d'école, détaillées dans deux textes officiels, le premier précisant les compétences professionnelles exigibles du professeur des écoles stagiaire à l'issue de sa formation (annexe lll à la note de service $n^{\circ}$ 94-271 du 16 novembre 1994), le second, celles du professeur des écoles en fonction (note de service $n^{\circ}$ 94-280 du 25 novembre 1994).

(2) Le recueil des données s'est effectué en 2001-2002 au centre départemental de Mâcon. Deux questionnaires ont été proposés aux stagiaires en début et fin d'année; ils les ont renseignés individuellement, sous le contrôle d'observateurs. Un sous-groupe de dix-sept PE2, aux caractéristiques - âge, sexe, études suivies, réponses aux questionnaires, etc. - délibérément diversifiées, a accepté en fin d'année un entretien semi-directif.

(3) Cf. Tardif, Lessard \& Gauthier, 1998. Les résultats de notre recherche ne confirment pas les conclusions de D. Michel (2001), selon laquelle les professeurs des écoles reconnaissent les savoirs théoriques comme utiles dans leur formation pour faire face aux situations professionnelles auxquelles ils seront confrontés. Selon cette chercheuse, $86 \%$ des PE2 interrogés ressentent un besoin de formation théorique. Les réponses des professeurs stagiaires des écoles, collèges et lycées interrogés par P. Rayou (2004) sont plus hétérogènes, puisque certains voudraient moins de théorie alors que d'autres en voudraient davantage. C'est qu'il convient d'être prudent quant aux significations que les professeurs stagiaires accordent à ce qui est "théorique" (on peut ne pas voir l'utilité concrète de savoirs théoriques, tout en escomptant tirer un profit au moins symbolique de l'affirmation de leur intérêt).

(4) En outre, ce souci paraît volatil dans notre échantillon : à ['exception de deux stagiaires, ce ne sont pas les mêmes qui expriment ces préoccupations dans les questionnaires de début et de fin d'année. L'épreuve de la pratique aurait-elle conduit les uns à relativiser leurs ambitions initiales et les autres, à en découvrir la valeur?
(5) A la lettre, douze occurrences ; mais aussi, sous des formulations équivalentes : être "submergé », «noyé », «nul»... : au total, dix-sept des soixante-dix sept stagiaires (la question posée dans le questionnaire de début d'année, était : " que redoutezvous de votre année de formation à l'IUFM en ce qui concerne les stages? ").

(6) Vingt-six stagiaires (un tiers de l'échantillon) appréhendent d'effectuer leur stage dans des classes difficiles et/ou de ne pas être respectés, de ne pas "faire face", d'être "débordés par les élèves». Certaines réponses, plus générales ou évasives, qui évoquent la "gestion de classe » ou "de groupes» (cinq), ou celle de la « diversité des élèves " (deux), relèvent peut-être des mêmes inquiétudes. On peut probablement y associer un certain nombre de ceux qui redoutent "de ne pas être à la hauteur", mais dissimulent derrière cette formule générale l'inquiétude très concrète de ne pas " tenir » leur classe.

(7) Quatre stagiaires sur dix déclarent redouter fortement soit la situation générale d'évaluation, avec le stress, voire la panique qu'elle pourrait susciter chez eux, soit le contenu de cette évaluation dont ils craignent qu'il soit " peu nuancé ", " démotivant ", "trop sévère", voire « destructeur ». vingt-trois stagiaires (un tiers de l'échantillon; ce sont parfois les mêmes que les précédents) sont inquiets des réponses concrètes que l'équipe de suivi du stage sera en mesure de leur apporter pour surmonter les difficultés rencontrées.

(8) Une réponse sur soixante-dix-sept en fait état.

(9) Seuls trois stagiaires (sur soixante-dix-sept) expriment leur crainte d'être démunis face à un élève en difficulté ; deux autres redoutent de faire des erreurs préjudiciables aux élèves, et un troisième craint « de ne rien apprendre aux élèves, leur faire perdre leur temps, les ennuyer "; deux autres réponses évoquent, plus généralement, la "responsabilité " à l'égard des enfants. 
(10) Delbos et Jorion (1984) différencient les savoirs et les modes de transmission des savoirs en deux types: formels et informels. Selon le mode formel, les savoirs se transmettent dans des institutions, lors de cours et de stages d'une formation organisée en vue de la qualification des stagiaires. Selon le mode informel savoirs et savoir-faire s'acquièrent dans le travail lui-même, comme l'un de ses « bénéfices secondaires".

(11) Lorsque c'est le fonctionnement même de l'école qui contredit les réglementations officielles, le stagiaire pourra s'appuyer sur la législation en vigueur et ne pas accepter le "réalisme " invoqué par les acteurs de terrain pour se défaire de leurs obligations. C'est beaucoup plus difficile lorsqu'en matière de pédagogie, ils font valoir une "expérience " dont le stagiaire est par définition dépourvu.

(12) Cf. Tolley, 2004. A partir de l'exemple de la formation des bergers sur le tas et en milieu institutionnel, $\mathrm{C}$. Tolley propose de relativiser l'opposition entre savoirs formels et informels : la formation sur le tas peut-être aussi prescriptive pour l'action (et donc portée par une intention formelle) que la formation institutionnelle; inversement, celle-ci s'accompagne souvent des "bénéfices secondaires" des transmissions informelles de savoirs et savoir-faire entre stagiaires.

(13) Cf. Beckers, 2004. Dans le centre de formation objet de l'enquête, et pour l'année considérée (2001-2002), cette « réflexion sur l'action " est pour l'essentiel constituée d'entretiens consécutifs aux visites des formateurs dans les classes de stagiaires, d'une journée de bilan en groupes restreints, lors d'un retour de stage (trois ou quatre dans l'année) et de la préparation du mémoire professionnel. Les formateurs du centre n'ayant bénéficié à cette date d'aucune formation particulière à l'analyse de la pratique, celle-ci semble entendue par eux dans une acception large, désignant des temps institutionnels d'évocation par les enseignants stagiaires, devant leurs pairs ou en relation duelle avec un formateur, de leur pratique professionnelle au cours des stages, éventuellement des plaisirs et souffrances qui l'ont accompagnée. Ces moments, au cours desquels les conseils des formateurs se combinent à la parole des stagiaires paraissent viser davantage l'amélioration de l'action ou le travai sur l'image de soi que la construction de compétences d'objectivation et d'analyse critique des pratiques.

(14) Par comparaison, trente-neuf réponses (sur soixante-dix-sept) valorisent, entre autres qualités, la relation proche aux enfants ou leur "écoute "; dix-neuf mettent en relief le dynamisme (ou la " motivation", l'enthousiasme,etc.), dix-sept l'autorité.

(15) Y. Saint-Arnaud (2001) oppose le « paradigme de l'incertitude " qui admet la part d'imprévisibilité dans l'interaction humaine et les limites vis-à-vis de celle-ci inhérentes à tout savoir homologué (théories, etc.), au "paradigme de l'expertise ", qui justifie l'inefficacité de l'action par une mauvaise application de ce savoir, les limites du public visé ou les contraintes de la situation.

(16) J.-C. Kaufmann rappelle que nos sociétés démocratiques ne peuvent promouvoir la liberté individuelle sans aménager en même temps divers cadres normalisateurs de la vie en société "L'affirmation officielle et explicite d'une liberté d'une part: chacun peut décider à son idée. Et parallèlement, la discrète mise en place de normes implicites du vivre ensemble » (2001, p. 239 ; cf. aussi Martuccelli, 2002).

(17) Ce que ne fait pas D. Martuccelli : "cette vision extrême, qu'i est difficile de partager jusqu'au bout... " (p. 517).

\section{RÉFÉRENCES BIBLIOGRAPHIQUES}

ALTET M. (1994). La formation professionnelle des enseignants. Paris : PUF.

Baillauquės S \& Breuse E. (1993). La première classe ou les débuts dans le métier d'enseignant. Paris : ESF.

BARRĖRE A. (2003). Travailler à l'école: que font les élèves et les enseignants du secondaire? Rennes: Presses universitaires de Rennes.

BECKERS J. (2004). « Comment amorcer la construction identitaire d'un praticien réflexif dans la formation initiale? ", Recherche et formation pour les professions de l'enseignement, $\mathrm{n}^{\circ} 46, \mathrm{p} .61-80$.

BLANCHARD-LAVILLE C. (2001). Les enseignants entre plaisir et souffrance. Paris : PUF.

BOURDONCLE R. (1991). " La professionnalisation des enseignants: analyses sociologiques anglaises et américaines ". Revue française de pédagogie, $n^{\circ} 94$, p. 73-91.

BOURDONCLE R. (1993). "La professionnalisation des enseignants: les limites d'un mythe ". Revue française de pédagogie, $n^{\circ} 105$, p. 83-119.

BOURDONCLE R. (2000). "Autour des mots : professionnalisation, formes et dispositifs", Recherche et formation pour les professions de l'éducation, $\mathrm{n}^{\circ} 35, \mathrm{p} .117-132$.

DAvISSE A. \& RocheX J.-Y. [dir.] (1997). "Pourvu qu'ils m'écoutent": discipline et autorité dans la classe. Mémoires professionnels d'enseignants-stagiaires (collège, lycée) à l'IUFM de Créteil. Créteil : CRDP de l'académie de Créteil.

Delbos G. \& Jorion P. (1984). La transmission des savoirs. Paris : Éd. de la MSH.
DUBET F. (2002). Le déclin de l'institution. Paris : Éd. du Seuil. Élias N. (1991). La société des individus. Paris : Fayard.

FoucAuLt M. (1975). Surveiller et punir : naissance de la prison. Paris: Gallimard.

GofFMAN E. (1973). La mise en scène de la vie quotidienne. Paris : Éd. de Minuit.

Hughes E. C. (1996). Le regard sociologique. Paris : Éd. de l'EHESS.

ISAMBERT-JAMATI V. (1985). "Les primaires, ces "incapables prétentieux" ", Revue française de pédagogie, $\mathrm{n}^{\circ} 73$, p. 57-67.

KaUfMANn J.-C. (2001). Ego: pour une sociologie de l'individu. Paris : Nathan.

LANG V. (1999). La professionnalisation des enseignants. Paris : PUF.

Malglaive G. (1990). Enseigner à des adultes. Paris : PUF.

Martuccelli D. (2002). Grammaires de l'individu. Paris: Gallimard.

MiCHEL D. (2001). « De l'instituteur au professeur des écoles: un changement de compétences mais aussi de formation: comment la formation théorique est-elle perçue par les professeurs des écoles stagiaires? ». Communication au colloque "Quelles compétences? Pour quelle professionnalisation ? ", Reims : 8-9 novembre 2001.

PerRenoud P. (1999). Dix nouvelles compétences pour enseigner. Paris : ESF.

Perrenoud P. (2001). Développer la pratique réflexive dans le métier d'enseignant. Paris : ESF. 
Peyronie H. (1998). Instituteurs : des maitres aux professeurs des écoles: formation, socialisation et "manière d'être au métier ". Paris : PUF.

RAYOu P. \& van ZANTEN A. (2004). Enquête sur les nouveaux enseignants : changeront-ils l'école. Paris : Bayard.

SAINT-ARNAUD Y. (2001). "La réflexion-dans-l'action, un changement de paradigme", Recherche et formation pour los profossions de l'enscignement, $\mathrm{n}^{\circ} 36, \mathrm{p} .17-27$.

ScHÖN D. (1983). The Reflective Practitioner, How Professionals Think in Action. New York : Basic Books [trad. fr. Le praticien réflexif : à la recherche du savoir caché dans l'agir professionnel. Montréal : Éd. Logiques, 1994].
TARdif M. ; Lessard C. \& Gauthier C. (1998). Formation des maitres et contextes sociaux. Paris : PUF.

TOLLEY C. (2004). « Formation scolaire ou formation sur le tas chez les bergers de Provence : différenciation des pratiques et conflit de légitimité ? ", Sociétés contemporaines, $\mathrm{n}^{\circ} 55, \mathrm{p} .115-138$.

Vermersch P. (1994). L'entretien d'explicitation. Paris: ESF.

VINCENT G. (1980). L'école primaire française. Lyon : Presses universitaires de Lyon.

VINCENT G. [dir.] (1994). L'éducation prisonnière de la forme scolaire? Scolarisation et socialisation dans les sociétés industrielles. Lyon: Presses universitaires de Lyon. 